\title{
Simulation of statistical fluctuations in the spin precession measurement at RHIC
}

\author{
A.A. Poblaguev
}

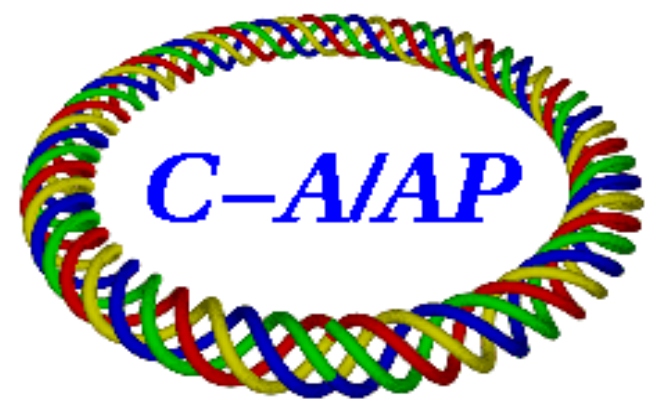

\section{Collider-Accelerator Department Brookhaven National Laboratory \\ Upton, NY 11973}

Notice: This document has been authorized by employees of Brookhaven Science Associates, LLC under Contract No. DE-AC02-98CH10886 with the U.S. Department of Energy. The United States Government retains a nonexclusive, paid-up, irrevocable, world-wide license to publish or reproduce the published form of this document, or allow others to do so, for United States Government purposes. 


\title{
Simulation of Statistical Fluctuations in the Spin Precession Measurements at RHIC
}

\author{
A.A. Poblaguev
}

February 25, 2014

\begin{abstract}
Measurements of the driven spin coherent precession $S_{x}(t)=S_{x}^{(0)}-S_{x}^{(1)} \sin \left(\omega t+\varphi_{0}\right)$ were initiated in RHIC Run13. The expected value of the precession amplitude $S_{x}^{(1)} \sim 2 \times 10^{-4}$ is about the statistical error in a single measurement and data fit gives a biased estimate of the $S_{x}^{(1)}$. For a proper statistical interpretation of the results of the several measurements, statistical fluctuations were studied using Monte-Carlo simulation. Preliminary results of the spin precession measurements in RHIC Run13 are presented.
\end{abstract}

\section{Introduction}

Proton beam polarization at the Relativistic Heavy Ion Collider (RHIC) is monitored by 4 pCarbon polarimeters [1]. Each polarimeter consist of 6 silicon detectors located as shown on Fig. 1. In a regular polarization measurement, the vertical component of the beam polarization $P_{y}$ is derived from the left/right asymmetry of the recoil Carbons production:

$$
A_{y}=\frac{N_{R}^{\uparrow}-N_{L}^{\uparrow}}{N_{R}^{\uparrow}+N_{L}^{\uparrow}}=\frac{N_{L}^{\downarrow}-N_{R}^{\downarrow}}{N_{L}^{\downarrow}+N_{R}^{\downarrow}}=P_{y}\left\langle A_{N}\right\rangle
$$

Here, $N_{L R}^{\uparrow \downarrow}$ is number of detected Carbons in the left/right detectors depending on the beam polarity. The average value of analyzing power $\left\langle A_{N}\right\rangle$ is calculated using predefined dependence $A_{N}(E)$ of analyzing power on Carbon kinetic energy and experimentally measured energy spectra of the Carbons. Measurements with flipping polarity of the beam allows one to strongly suppress systematic errors in the measurements:

$$
A_{y}=\frac{\sqrt{N_{R}^{\uparrow} N_{L}^{\downarrow}}-\sqrt{N_{L}^{\uparrow} N_{R}^{\downarrow}}}{\sqrt{N_{R}^{\uparrow} N_{L}^{\downarrow}}+\sqrt{N_{L}^{\uparrow} N_{R}^{\downarrow}}}
$$

This solution is usually referred as "the square root formula". For small values of intensity $(\uparrow \downarrow)$, acceptance $(L R)$ and physical $\left(A_{y}\right)$ asymmetries, the statistical accuracy of the determination of the asymmetry depends only on the total statistics of the measurement:

$$
\sigma_{A} \approx \sqrt{\frac{1}{N_{\mathrm{tot}}}}=\sqrt{\frac{1}{N_{R}^{\uparrow}+N_{L}^{\downarrow}+N_{L}^{\uparrow}+N_{R}^{\downarrow}}}
$$




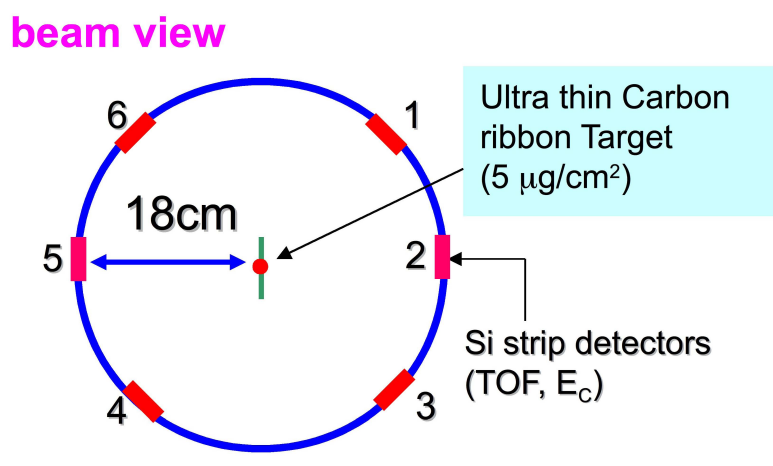

Figure 1: A schematic view of the RHIC pCarbon Polarimeter. Each detector consists of 12 Si strips. Recoiled Carbon signals are isolated by comparison of measured Carbon time of flight and energy deposited in the strip.

In RHIC Run13 the study of the driven spin coherent precession [2] was attempted. The precession may be identified by observing oscillation amplitude $S_{x}^{(1)}$ of the horizontal component of the beam proton spin (perpendicular to the beam direction).

$$
S_{x}(t)=S_{x}^{(0)}-S_{x}^{(1)} \sin \left(\omega t+\varphi_{0}\right)
$$

The oscillation frequency $\omega$ is controlled by the AC Dipole [3] and is known with a very high precision. One can determine the horizontal component of the beam polarization by measuring up/down asymmetry of recoil Carbons production:

$$
A_{x}=\frac{\sqrt{N_{U}^{\uparrow} N_{D}^{\downarrow}}-\sqrt{N_{D}^{\uparrow} N_{U}^{\downarrow}}}{\sqrt{N_{U}^{\uparrow} N_{D}^{\downarrow}}+\sqrt{N_{D}^{\uparrow} N_{U}^{\downarrow}}}
$$

In this case only detectors 1, 6 (up) and 3,4 (down) are employed. The spin precessing around stable spin direction ${ }^{1}$ results in oscillation of measured horizontal asymmetry:

$$
A_{x} \times 10^{3}=a_{0}+a \sin \left(\omega t+\varphi_{0}\right)
$$

The factor $10^{3}$ is entered here to define the scale of the asymmetry amplitudes $a_{0}, a \sim 1$ used in this note.

The results of one such measurement [4] (RHIC run 17597.006) is shown on Fig. 2. The total statistics in this measurement was $51.3 \times 10^{6}$ events. For every event, the phase $\varphi(0 \leq \varphi<2 \pi)$ was derived from the event time $t$ counted relative to a special phase marker signal. The asymmetry was calculated separately for each of 8 equal phase bins.

\section{Results dependence on the number of bins.}

Generally, a small number of bins used in data analysis may result in systematic error in the measurement. To evaluate this effect, $10^{9}$ events in the polarimeter(factor 20 larger

\footnotetext{
${ }^{1}$ This direction has a few degree deviation from the vertical line.
} 


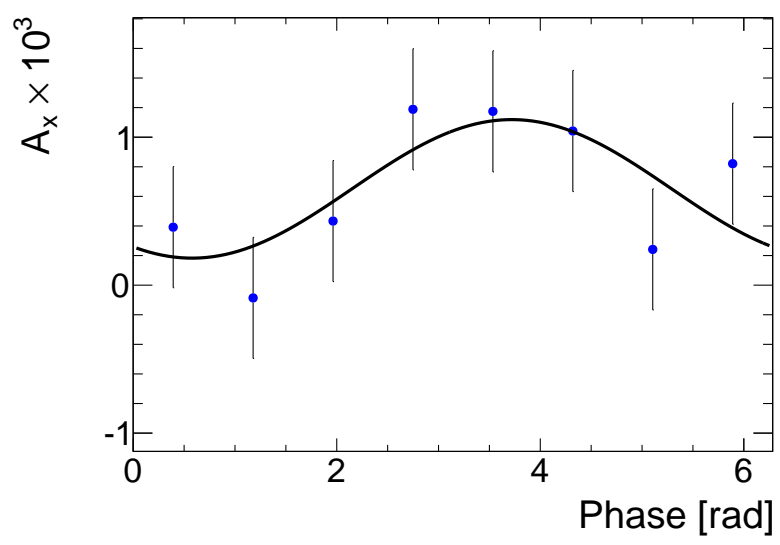

Figure 2: A single run (17597.006) data fit: $a_{0}=0.65 \pm 0.14, a=0.47 \pm 0.20, \varphi_{0}=2.56 \pm 0.44$.

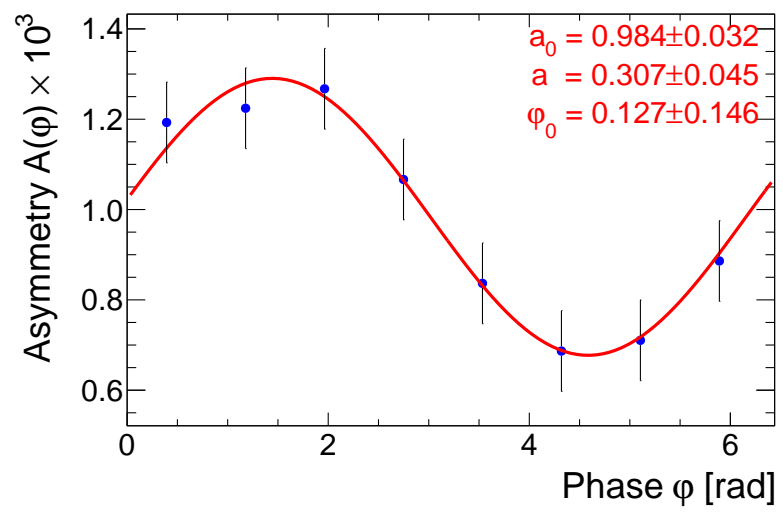

Figure 3: Simulation of the precessing amplitude measurement with $10^{9}$ events.

than in a regular measurement at RHIC) was simulated. In the simulation, events were distributed between up and down detectors in accordance with beam polarity and spin precessing phase. The following asymmetry oscillation parameters were used

$$
a_{0}=1.0, \quad a=0.3, \quad \varphi_{0}=0 .
$$

The result of the simulation is displayed in Fig. 3. The evaluated precessing amplitude $a$ is consistent within a $15 \%$ statistical error with a value of 0.3 used in the simulation.

Statistical sensitivity to the number of bins may be enormously increased with no change of relative systematic error if we just enlarge the value of $a$ by a factor of 100. Simulation of $200 \mathrm{M}$ events with such a parametrization allowed us to evaluate systematic error due to the number of bins $n_{b}$ with high accuracy

$$
\delta a_{\text {syst }} / a= \begin{cases}-10.0 \% & \text { if } n_{b}=4 \\ -2.6 \% & \text { if } n_{b}=8 \\ -0.7 \% & \text { if } n_{b}=16\end{cases}
$$


Thus, for any reasonable statistics of the measurements at RHIC, the systematic error due the number of bins $n_{b}=8$ is negligible compared to the statistical error.

The systematic errors shown in Eq. (8) can be reproduced in a simple calculation. If the value of phase corresponding to the bin $i$ center is $\varphi_{i}$ and the bin width is $2 \delta=2 \pi / n_{b}$ than the sinusoid amplitude will be averaged as

$$
\sin \left(\varphi_{i}+\varphi_{0}\right) \Rightarrow \frac{1}{2 \delta} \int_{\varphi_{i}-\delta}^{\varphi_{i}+\delta} \sin \left(\varphi+\varphi_{0}\right) d \varphi=\sin \left(\varphi_{i}+\varphi_{0}\right) \frac{\sin \delta}{\delta}
$$

Thus, the binning results in scaling of the precession amplitude by a factor

$$
\frac{\sin \left(\pi / n_{b}\right)}{\pi / n_{b}} \approx 1-\frac{\pi^{2}}{6 n_{b}^{2}}
$$

The calculated scaling factor (10) is in a good agreement with results of simulation $(8)$.

\section{Simulation of the results of a single measure- ments.}

To study the effects of statistical fluctuations in the precession measurement it is convenient to use the following parametrization of the asymmetry oscillation

$$
a(t)=a_{0}+a_{c} \sin \omega t+a_{s} \cos \omega t
$$

which is derived from the Eq. (6) by substituting $a_{c}=a \cos \varphi_{0}$ and $a_{s}=a \sin \varphi_{0}$.

The event distribution over the measured phase $\omega t+\varphi_{0} \rightarrow \varphi(0 \leq \varphi<2 \pi)$ may be parametrized as

$$
d N / d \varphi=N_{0}\left(1+a_{0}+a_{c} \sin \varphi+a_{s} \cos \varphi\right)
$$

For simplicity, four measured numbers of events $N_{U D}^{\uparrow \downarrow}$ (see Eq. (5)) were replaced by one number $N$ assuming that $N_{0}$ is known. ${ }^{2}$ For such a distribution, the parameters $a_{0}, a_{c}$, and $a_{s}$ can be measured as:

$$
\begin{aligned}
N_{0} a_{0} & =\int N(\varphi) d \varphi-N_{0} \\
N_{0} a_{c} & =\int 2 \sin \varphi N(\varphi) d \varphi \\
N_{0} a_{s} & =\int 2 \cos \varphi N(\varphi) d \varphi
\end{aligned}
$$

It directly follows from Eqs. (13-15) that statistical errors

$$
\sigma_{0}=\sqrt{1 / N}, \quad \sigma_{c}=\sigma_{s}=\sqrt{2 / N}
$$

\footnotetext{
${ }^{2}$ The validity of such an approximation for the statistical error estimate is implicitly provided by the square root formula.
} 


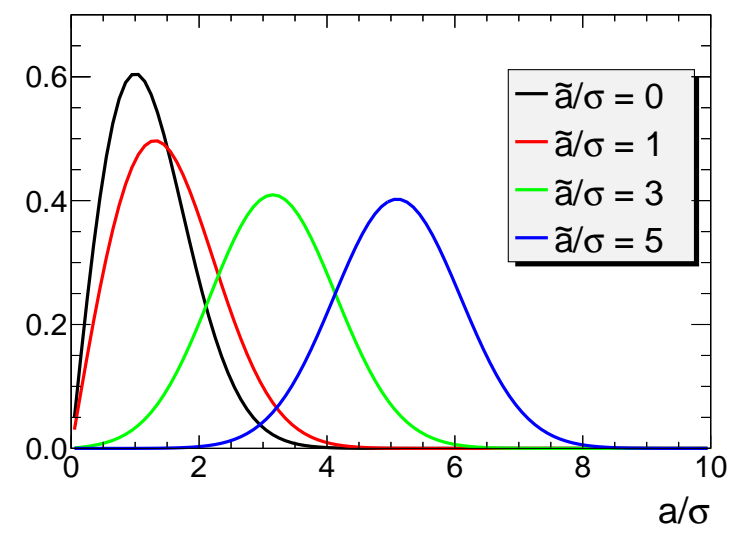

Figure 4: Normalized distributions of the measured precession amplitude $a$.

of the determination of parameters $a_{0}, a_{c}$, and $a_{s}$, respectively, are unequivocally derived from the total statistics $N$ of the measurement. Also the statistical fluctuations for these parameters are uncorrelated

$$
\left\langle\delta_{0} \delta_{c}\right\rangle=\left\langle\delta_{0} \delta_{s}\right\rangle=\left\langle\delta_{c} \delta_{s}\right\rangle=0
$$

The simplest way to simulate the result of a single measurement of spin precession is just to assign Gaussian distributed random numbers $G($ mean, $\sigma$ ) to the measured parameters:

$$
\begin{aligned}
& a_{0}=G\left(\tilde{a}_{0}, \sqrt{1 / N}\right) \\
& a_{c}=G\left(\tilde{a}_{c}, \sqrt{2 / N}\right) \\
& a_{s}=G\left(\tilde{a}_{s}, \sqrt{2 / N}\right)
\end{aligned}
$$

Here, $N$ is total number of events in the measurement and $\tilde{a}_{0}, \tilde{a}_{c}$, and $\tilde{a}_{s}$ are actual values of the spin precessing parameters. With no loss of generality we may assume

$$
\tilde{a}_{s}=0, \quad \tilde{a}_{c}=\tilde{a}
$$

where $\tilde{a}$ is the precession amplitude. For the RHIC fill 17597 measurements $(N \sim 51 M$ events), the statistical errors for the precession parameters $a_{c}$ and $a_{s}$ are equal to

$$
\sigma=0.20 \text {. }
$$

Expected distributions of measured precession amplitude $a$ for several values of $\tilde{a}$ are shown on Fig. 4. Taking into account the distribution dependence on $\tilde{a}$, the result of data fit $a_{17599.006}^{\text {(fit) }}=0.47 \pm 0.20$ (Fig. 2) should be statistically interpreted as:

$$
a_{17599.006}=0.42_{-0.22}^{+0.21}
$$

For analysis, it is convenient to consider the square of the precessing amplitude:

$$
\begin{aligned}
a^{2} & =\tilde{a}^{2}+2 \tilde{a} \sigma \delta_{c}+\sigma^{2}\left(\delta_{c}^{2}+\delta_{s}^{2}\right) \\
& =\left(\tilde{\rho}+2 \sqrt{\tilde{\rho}} \delta_{c}+\delta_{c}^{2}+\delta_{s}^{2}\right) \sigma^{2}
\end{aligned}
$$


Here $\tilde{\rho}=\tilde{a}^{2} / \sigma^{2}$ is a parametrization of the actual spin precession amplitude $\tilde{a}$ and $\delta_{c, s}=G(0,1)$ are Gaussian distributed random numbers. The mean measured value of $a^{2}$ and variance may be evaluated as

$$
\left\langle a^{2}\right\rangle=(\tilde{\rho}+2) \sigma^{2} \quad \sigma_{a^{2}}^{2}=4(\tilde{\rho}+1) \sigma^{2}
$$

One can see that measured value of $a^{2}$ gives a biased (over-evaluated) estimate of the $\tilde{a}^{2}$. The bias becomes negligible if $a \gg \sigma$. Since the bias, $2 \sigma^{2}=4 / N$, is unambiguously calculated from the total number of events $N$ in the measurement, we can employ the unbiased estimate $\rho$ of the spin precession parameter $\tilde{\rho}$ :

$$
\rho=a^{2} / \sigma^{2}-2=\tilde{\rho}+2 \sqrt{\tilde{\rho}} \delta_{c}+\delta_{c}^{2}+\delta_{s}^{2}-2
$$

Mathematical expectation and variance of the measured parameter $\rho$ are equal to

$$
\langle\rho\rangle=\tilde{\rho}, \quad \sigma_{\rho}=2 \sqrt{1+\tilde{\rho}},
$$

respectively.

In terms of parameter $\rho$, the results of run 17597.006 may be presented as

$$
\rho_{17597.006}=3.52
$$

\section{How to combine several measurements.}

The expected value of precession amplitude in RHIC fill 17599 is about $\tilde{a} \approx 0.2$ [5]. It can not be reliably isolated in a single measurement with statistical accuracy of $\sigma \approx 0.20$.

If there are $n$ measurements and relative phase in the measurements is known (coherent measurements), then we can fit all data together. The statistical fluctuations of such a combined measurement will be described by the same equation (24) after a substitution $\sigma \rightarrow \sigma / \sqrt{n}$. In this case:

$$
\rho^{c o h}=a^{2} / \sigma^{2}-2 / n, \quad \sigma_{\rho}^{c o h}=\frac{2 \sqrt{\tilde{\rho}+1 / n}}{\sqrt{n}}
$$

Here $a$ is amplitude measured in the combined fit.

Alternatively, we can measure (after proper alignment of the phase) amplitudes $a_{c}^{(i)}$ and $a_{s}^{(i)}$ in every run $i$ separately and than calculate the average precession amplitude

$$
a^{2}=a_{c o h}^{2}=\left(\frac{\sum a_{c}^{(i)}}{n}\right)^{2}+\left(\frac{\sum a_{s}^{(i)}}{n}\right)^{2}
$$

which should be used in Eq. (29).

If phases in the measurements are not aligned (incoherent measurements) then we can calculate the average value

$$
\bar{a}^{2}=a_{i n c}^{2}=\frac{\sum\left(a_{c(i)}^{2}+a_{c(i)}^{2}\right)}{n}
$$



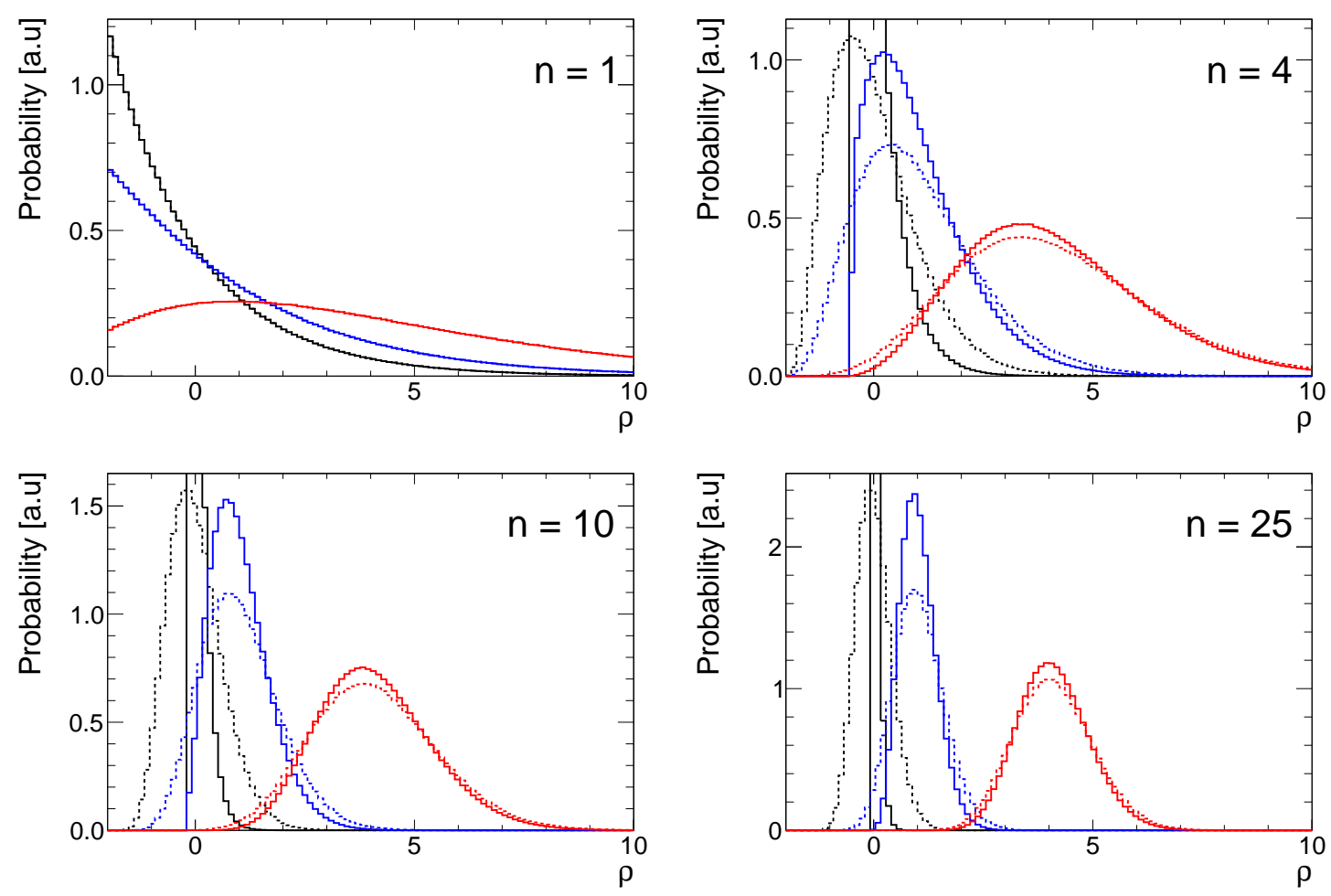

Figure 5: Distributions of unbiased estimates $\rho$ of the precessing amplitude $\tilde{a}$ depending on number of measurements $n$ and actual value of $\tilde{a}=0$ (black lines), $\tilde{a}=0.2$ (blue lines), $\tilde{a}=0.4$ (red lines). Solid lines correspond to the coherent measurements while dashed lines are for incoherent measurements. The statistical fluctuations in a single measurement was assumed to be $\sigma=0.20$ (51M events).

In this case

$$
\rho^{i n c}=\bar{a}^{2} / \sigma^{2}-2, \quad \sigma_{\rho}^{i n c}=\frac{2 \sqrt{\tilde{\rho}+1}}{\sqrt{n}}
$$

Obviously, coherent measurements provide better accuracy of determination of precession amplitude $\tilde{a}$. However, for a relatively large signal $\tilde{a} \gtrsim \sigma$ effect is not essential.

Using Eq. (24) one can find the distribution of measured values of $\rho$ for any assumed value of the actual precessing amplitude $\tilde{a}$ and number of measurements $n$. Such distributions of $\rho$ for several values of $\tilde{\rho}$ and $n$ are shown on Fig. 5 .

\section{Confidence intervals}

These distributions may be used to determine confidence intervals for the measured value of $\rho$ (or $a$ ). If $\mathcal{P}_{n}(\rho, \tilde{\rho})$ is a probability that unbiased estimate of precession amplitude will be larger than $\rho$ in a set of $n$ measurements of the actual value of $\tilde{\rho}$, then for any given probability $p$ and measured value of $\rho$ one can find the corresponding 
value of $\tilde{\rho}$ :

$$
p=\mathcal{P}_{n}\left(\rho, \tilde{\rho}=\rho_{p}\right) \quad \Rightarrow \quad \rho_{p}=\mathcal{R}_{n}(\rho, p)
$$

Equation (33) allows one to present the results of measurement of precessing amplitude in a standard way:

$$
a_{\text {meas }}^{2} / \sigma^{2}=\left(\rho_{0.5}\right)_{-\sigma_{-}}^{+\sigma_{+}}
$$

where $\sigma_{+}=\rho_{1-\alpha / 2}-\rho_{0.5}, \sigma_{-}=\rho_{0.5}-\rho_{\alpha / 2}$, and $1-\alpha$ is a confidence interval. For standard errors $(1 \sigma)$ the value of $\alpha=0.3173$ should be used.

If $\rho_{\alpha / 2}>0$ the result of the measurement may be rewritten as

$$
a_{\text {meas }}=\left(\sigma \sqrt{\rho_{0.5}}\right)_{-\left(\sqrt{\rho_{0.5}}-\sqrt{\rho_{\alpha / 2}}\right) \sigma}^{+\left(\sqrt{\rho_{0.5}}\right) \sigma},
$$

otherwise the upper limit should be set:

$$
a_{\text {meas }}<\sigma \sqrt{\rho_{\alpha / 2}}(1-\alpha \mathrm{CL})
$$

\section{Precession measurements in RHIC Run13}

In the RHIC Run13, there were totally 10 measurements of spin precessing amplitude. All data may be separated into 3 groups with slightly different condition of measurements. Every measurement took about $30 \mathrm{sec}$ with about $5 \mathrm{~min}$ interval between measurement in the same group. The relative phase in every group of measurements was controlled by sending a phase marker signal.

The preliminary results of data analysis are displayed in Fig 6 and Table 1. Only results of the second group of measurements (runs 17597.006-008) may be interpreted as observation of the precessing amplitude. However, confidence level of such an observation is not sufficient. On other hand, there is no disagreement within statistical errors between results of the measurements and expected values of the precessing amplitude.
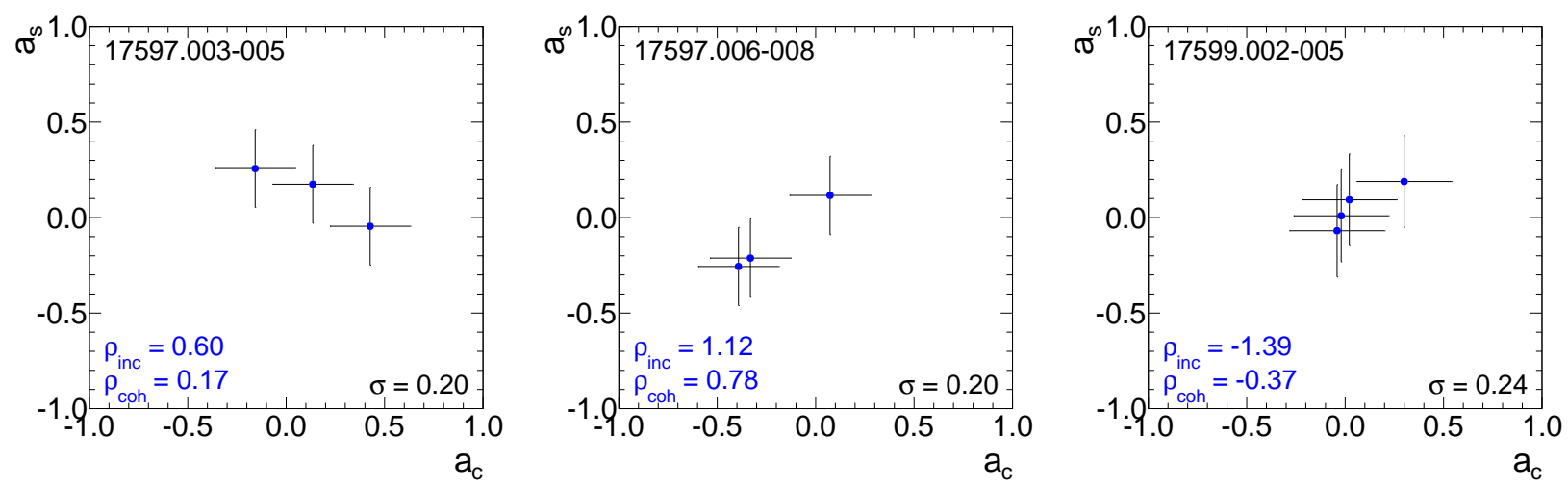

Figure 6: Measurements of the precessing amplitude in RHIC Run13. 


\begin{tabular}{l|c|cc|cc} 
& Expected & \multicolumn{2}{|c|}{ Incoherent } & \multicolumn{2}{|c}{ Coherent } \\
\cline { 3 - 6 } RHIC runs & $a$ & $\operatorname{Prob}(a>0)$ & $a$ & $\operatorname{Prob}(a>0)$ & $a$ \\
\hline $17597.003-005$ & 0.15 & $63 \%$ & $<0.28(90 \%$ CL $)$ & $75 \%$ & $<0.37(90 \%$ CL) \\
$17597.006-008$ & 0.18 & $85 \%$ & $0.24_{-0.21}^{+0.14}$ & $85 \%$ & $0.19_{-0.16}^{+0.12}$ \\
$17599.002-005$ & 0.15 & $4 \%$ & $0.0(90 \%$ CL $)$ & $23 \%$ & $<0.16(90 \%$ CL)
\end{tabular}

Table 1: Preliminary results of the statistical analysis of the precession amplitude measurements. Results are shown separately for coherent and incoherent phases in the measurements. The expected precession amplitudes are given in accordance with [5]. The $\operatorname{Prob}(a>0)=1-\mathcal{P}_{n}\left(\rho_{\text {meas }}, 0\right)$ is probability that a non-zero precessing amplitude was observed.

\section{Summary}

In this note, a method of simulation of the statistical fluctuations in the spin precessing measurements at RHIC was discussed.

It was shown that binning of signal phases leads to the well controlled systematic error which is negligible compared to the statistical error of measurements.

It was found that precessing amplitude determined in the data fit is a biased (overevaluated) value of the actual amplitude. The methods of calculation of the unbiased amplitude both for coherent and incoherent measurements as well as ways of statistical analysis of results of measurement was discussed.

Even though the preliminary analysis has not yielded any statistically significant measurement of driven spin precession, the second line in Table 1 indicates that the precession may be isolated with more data acquired.

\section{Acknowledgments}

The driven spin coherent precession measurements in RHIC Run13 had been prformed by M. Bai, J. Kewisch, P. Oddo, and D. Smirnov. I would like to thank them for helpful and stimulating discussions on the subject of this note.

\section{References}

[1] A. Bazilevskiy, et al, Precision measurements of the proton beam absolute polarization in RHIC, AIP Conf. Proc. 1149 (2009) 723.

[2] M. Bai, T. Roser, G. Bunce and R. Gill, "Driven spin coherent precession at RHIC," AIP Conf. Proc. 1149, 817 (2009).

[3] M. Bai et al, "RHIC AC Dipole Design and Construction," Proc. 2001 IEEE U.S. Part. Accel. Conf.

[4] RHIC Polarimetry Results: p-Carbon. http://www.phy.bnl.gov/cnipol/rundb/

[5] M. Bai, private communication. 